\title{
Possíveis fatores de risco para o desenvolvimento em crianças com cardiopatia congênita ${ }^{123}$
}

\author{
Possible risk factors for the development of children with congenital heart disease \\ Posibles factores de riesgo para el desarrollo de niños con cardíacas congénitas
}

Recebido: 06/08/2021 | Revisado: 11/08/2021 | Aceito: 23/08/2021 | Publicado: 24/08/2021
Regina Maria Ayres de Camargo Freire ORCID: https://orcid.org/0000-0002-6116-6165 Pontifícia Universidade Católica São Paulo, Brasil E-mail: freireregina@uol.com.br
Karine de Paula Tressoldi ORCID: https://orcid.org/0000-0001-7176-0486 Pontifícia Universidade Católica São Paulo, Brasil E-mail: karineptressoldi@gmail.com
Rafaela Joaquim Frizzo
ORCID: https://orcid.org/000-0002-6463-7524 Pontifícia Universidade Católica São Paulo, Brasil E-mail: rafaelajfrizzo@hotmail.com
Juliana de Souza Moraes Mori
ORCID: https://orcid.org/000-0002-9804-4874 Pontifícia Universidade Católica São Paulo, Brasil E-mail: jsmmori@hotmail.com
Daniela Iudice Rafael
ORCID: https://orcid.org/0000-0003-4363-4807 Pontifícia Universidade Católica São Paulo, Brasil E-mail: emptio_spai@hotmail.com
Julia Marques Pinto
ORCID: https://orcid.org/000-0002-3120-6154 Pontifícia Universidade Católica São Paulo, Brasil E-mail: julia.marques.pinto@gmail.com
Larissa Amorim
ORCID: https://orcid.org/0000-0003-4925-6480 Pontifícia Universidade Católica São Paulo, Brasil E-mail: larissa.amorimd@hotmail.com

\begin{abstract}
Resumo
Objetivo: Investigar a correlação entre fatores sociodemográficos, cirúrgicos, nosológicos e o desenvolvimento neuropsicomotor e de linguagem em crianças com cardiopatia congênita e observar os efeitos da intervenção multidisciplinar no desenvolvimento destas crianças. Método: estudo descritivo, experimental e longitudinal de crianças com idades entre 10 meses e 9 anos com cardiopatia congênita que foram avaliadas em relação ao desenvolvimento motor, cognitivo e de linguagem e acompanhadas por um período de até um ano. Os dados sócios demográficos e sobre o histórico do desenvolvimento neuropsicomotor foram obtidos a partir de entrevista com a mãe ou cuidadores e os dados cirúrgicos e nosológicos foram extraídos dos prontuários do hospital em que elas foram operadas e vem sendo acompanhadas desde o nascimento. Resultados: Não se observou correlação de qualquer natureza entre tempo de internação e desenvolvimento posterior da criança. A correlação tempo de internação $\mathrm{x}$ resultados dos Testes PROC e CDC não apresentaram significância, ou seja, as 07 crianças analisadas não apresentaram resultados discrepantes quando comparadas entre si ou entre si e o tempo maior ou menor de internação. Também não se encontrou correlação entre o desenvolvimento ao longo do ano e o tempo de internação ou o número de cirurgias. Observa-se que todas as crianças têm uma condição adversa na linguagem. Pode-se afirmar que a maioria avançou desde onde estava quando avaliada, após a intervenção proposta, sustentando a importância de um tratamento multidisciplinar. Conclusão: Constatou-se, também, a importância da intervenção multidisciplinar no desenvolvimento destas crianças e, na consequente, melhoria da qualidade de vida.
\end{abstract}

Palavras-chave: Cardiopatia infantil; Desenvolvimento infantil; Reabilitação; Linguagem; Fonoaudiologia.

\footnotetext{
${ }^{1}$ Este artigo é parte do projeto de pesquisa 'Acompanhando o desenvolvimento da linguagem e suas relações em crianças com cardiopatia

congênita', coordenado por Cristiane Pessotti Ximenes e desenvolvido no HCor em parceria com a PUC-SP.

${ }^{2}$ Nossos agradecimentos a Marcel Amaral Marques Ferraz e Pauline Louise Herbach por suas contribuições ao trabalho realizado no ambulatório de pediatria do HCor.

${ }^{3}$ Agradecimentos ainda e, principalmente, ao CNPq e à CAPES pelos auxílios concedidos.
} 


\begin{abstract}
Objective: To investigate the correlation between sociodemographic, surgical, nosological factors and neuropsychomotor and language development in children with congenital heart disease and to observe the effects of multidisciplinary intervention on the development of these children. Method: descriptive, experimental, and longitudinal study of children aged between 10 months and 9 years with congenital heart disease who were evaluated in relation to motor, cognitive and language development and followed up for a period of up to one year. The sociodemographic data and the history of neuropsychomotor development were obtained from an interview with the mother or caregivers, and the surgical and nosological data were extracted from the hospital records where they were operated and have been followed up since birth. Results: There was no correlation of any kind between length of stay and later development of the child. The correlation length of hospital stays x results of the PROC and CDC Tests was not significant, that is, the 07 children analyzed did not present discrepant results when compared to each other or to each other and the longer or shorter length of stay. There was also no correlation between development over the year and length of stay or number of surgeries. It is observed that all children have an adverse language condition. It can be said that most advanced from where they were when evaluated, after the proposed intervention, sustaining the importance of a multidisciplinary treatment. Conclusion: It was also noted the importance of multidisciplinary intervention in the development of these children and, consequently, improving their quality of life.
\end{abstract}

Keywords: Childhood heart disease; Child development; Rehabilitation; Language; Speech therapy.

\title{
Resumen
}

Objetivo: Investigar la correlación entre factores sociodemográficos, quirúrgicos, nosológicos y el desarrollo neuropsicomotor y del lenguaje en niños con cardiopatías congénitas y observar los efectos de la intervención multidisciplinaria en el desarrollo de estos niños. Método: estudio descriptivo, experimental y longitudinal de niños de entre 10 meses y 9 años con cardiopatías congénitas que fueron evaluados con relación al desarrollo motor, cognitivo y del lenguaje y seguidos por un período de hasta un año. Los datos sociodemográficos y los antecedentes de desarrollo neuropsicomotor se obtuvieron de una entrevista con la madre o cuidadores, y los datos quirúrgicos y nosológicos se extrajeron de los registros hospitalarios donde fueron intervenidos y se les ha dado seguimiento desde su nacimiento. Resultados: No hubo correlación de ningún tipo entre la duración de la estadía y el desarrollo posterior del niño. La correlación tiempo de estancia hospitalaria x resultados de las Pruebas PROC y CDC no fue significativa, es decir, los 07 niños analizados no presentaron resultados discrepantes cuando se compararon entre sí o entre sí y la estancia más larga o corta. Tampoco hubo correlación entre el desarrollo a lo largo del año y la duración de la estadía o el número de cirugías. Se observa que todos los niños tienen una condición de lenguaje adversa. Se puede decir que los más avanzados desde donde se encontraban cuando fueron evaluados, luego de la intervención propuesta, sustentando la importancia de un tratamiento multidisciplinario. Conclusión: También se señaló la importancia de la intervención multidisciplinar en el desarrollo de estos niños y, en consecuencia, en la mejora de su calidad de vida. Palabras clave: Enfermedad cardíaca infantil; Desarrollo del niño; Rehabilitación; Lenguaje; Terapia del lenguaje.

\section{Introdução}

A cardiopatia congênita é a principal anormalidade em neonatos, sendo seis vezes mais comuns que as anomalias cromossômicas e quatro vezes mais comuns que os defeitos do tubo neural (Oster, Lee, Honien, Riehle-Colarusso, Shin, Correa, 2013; Licht, Shera, Clancy, et al.,2009). Nos Estados Unidos, a prevalência de cardiopatia congênita está em torno de 1 a cada 110 nascidos vivos, sendo que $25 \%$ dos casos compreendem um grupo conhecido como defeitos cardíacos congênitos críticos com necessidade de intervenção na fase neonatal ou na primeira infância (Licht, Wang, Silvestre, Nicolson, Montenegro, Wernovsky, et al.,2004).

Dentre as cardiopatias congênitas conhecidas, uma das principais e mais graves é a chamada de coração univentricular que pode ser identificada pela existência de um ventrículo morfologicamente único, que pode ser o esquerdo ou o direito (Andropoulos, Hunter, Nelson, Stayer, Stark, McKenzie, et al.,2010). Frequentemente, a cardiopatia congênita complexa do tipo coração univentricular está associada com a transposição de grandes vasos e estenose pulmonar e o tratamento mais efetivo é a cirurgia - três no total --, realizadas até o primeiro ano de vida (Beca, Gunn, Coleman, Hope, Reed, Hunt, et al.,2013). Atualmente, com os aprimoramentos do diagnóstico fetal, intervenção precoce, técnicas cirúrgicas e cuidados perioperatórios, cerca de 90\% dos cardiopatas atingem a idade adulta (Licht, Wang, Silvestre, Nicolson, Montenegro, Wernovsky, et al., 2004). Associadas à redução na mortalidade, as inabilidades por comprometimento do neurodesenvolvimento vêm sendo mais frequentemente reconhecidas. 
As crianças que nascem com cardiopatias graves, que demandam cirurgia, não uma, mas uma série delas, entram, imediatamente, em situação de risco para seu desenvolvimento geral (Cunha, Silva, Palladino, 2006). Risco que implica não apenas sua sobrevivência, mas, também, seu crescimento físico, psíquico e social, por dois motivos básicos: o perigo excepcional da doença e as diversas e seguidas hospitalizações, sobretudo em UTI, o que dificulta uma interação da criança com seus familiares e seu meio ambiente, comprometendo suas experiências e desenvolvimento.

Existe, atualmente, um crescente interesse em pesquisas sobre morbidade e qualidade de vida de crianças com cardiopatias congênitas (Hövels-Gürich, 2012,2016). Estudos sobre o desenvolvimento de crianças com cardiopatia congênita geralmente apontam para déficits no desenvolvimento motor, cognitivo e de linguagem (Gaynor, Stopp, Wypij, Andropoulos, Atallah, Atz, et al., 2015; Marino, Lipkin, Newburger, Peacock, Gerdes, Gaynor, et al., 2012). Embora atrasos no desenvolvimento tendem a diminuir com o tempo (Hövels-Gürich,2016b) eles ainda são observados muitos anos após a cirurgia bem-sucedida e potencialmente se manifestam em dificuldades de aprendizagem ou comportamentais (Newburger, Jonas, Wernovsky, Wypij, Hickey, Kuban, et al.,1993) o que implica que eles não são transitórios por natureza.

Uma lacuna identificada nas pesquisas é o papel dos preditores ambientais para o desenvolvimento de crianças com cardiopatia congênita, como a relação entre cuidador primário e criança. Estudos indicam a relevância da qualidade da parentalidade para o desenvolvimento em grupos clinicamente vulneráveis ${ }^{1}$ (Rappaport, Wypij, Bellinger, Helmers, Holmes, Barnes, et al.1998). O bebê que sofre de cardiopatia congênita precisa ser imediatamente acolhido pela tutela de um adulto (Jerusalinsky, 2004). Este acolhimento se efetiva nas condutas do adulto em relação ao bebê, que são, basicamente, condutas de cuidado.

Tal efetividade será alcançada não apenas pelos cuidados oferecidos a ele, mas, sobretudo, pelas palavras com as quais o adulto embala seus gestos cuidadores, já que este conjunto é que vai lhe conferir existência e lhe assegurar afeto. Falar com a criança de forma afetiva, cantar, fazer uso de elementos prosódicos, são gestos que, ao lado dos cuidados necessários, dão consistência a este acolhimento.

Assim, é uma urgência com o bebê portador de cardiopatia grave, tratar das suas interações com o outro, sejam seus pais sejam os profissionais da equipe responsável devido aos possíveis impactos no seu desenvolvimento, a fim de minimizar os efeitos negativos decorrentes de sua história de internações, cirurgias e intervenções esperadas pela cardiopatia.

Preocupado com tais adversidades, um grupo de pesquisadores resolveu enfrentar o desafio de intervir junto a crianças cardiopatas, com uma equipe multidisciplinar em razão dos vários aspectos do desenvolvimento afetados tanto pela cardiopatia como por suas implicações correlatas. Para dar início à intervenção, propôs-se uma avaliação inicial para traçar um planejamento de trabalho e tomou-se como objetivo investigar a correlação entre fatores sociodemográficos, cirúrgicos e nosológicos e o desenvolvimento de linguagem em crianças com cardiopatia congênita e observar os efeitos da intervenção multidisciplinar no desenvolvimento destas crianças.

\subsection{Revisão de literatura}

Um grande número de pesquisadores (Mari, Cascudo, Alchieri,2016; Mari, Alchieri,2012; Bertoletti, Marx, Júnior Hattge, Pellanda,2014; Snookes, Gunn, Eldridge, Donath, Hunt, Galea, et al.,2010) tem defendido que os efeitos da cardiopatia congênita se manifestam de maneira singular no desenvolvimento de cada criança; algumas terão déficits motores enquanto outras apresentarão apenas alterações cognitivas e/ou intelectuais e o mesmo ocorrendo nas outras áreas: social, afetiva e de linguagem.

Pode-se afirmar que a cardiopatia congênita afeta não só as crianças, mas também a sua família, a qual irá se mobilizar em função da nova condição que a doença ocasiona (Dos Santos, Dias, Salimena, Bara,2011). 
Então, para que possamos entender melhor essa temática, traremos estudos que articulem a qualidade de vida a quadros de cardiopatia infantil e estudos que abordem o desenvolvimento geral da criança.

a) Qualidade de vida

Alguns artigos afirmam que a qualidade de vida da criança cardiopata e sua família sofre as interferências desse quadro nosológico devido a: as inúmeras internações, o número de cirurgias, a superproteção dos pais, o estigma da doença, as restrições às atividades de alto impacto e a proximidade com a morte ${ }^{1}$ Leal, Silva da, Aita, Monteiro, de Montalvão,2016; Barreto, Sakamoto, Magagnin, Coelho, Waterkemper, Canabarro, 2016; Aita, De Souza,2016).

Por outro lado, (Krol, Grootenhuis, Destrée-Vonk, Lubbers, Koopman, Last, 2003) afirmam que crianças cardiopatas apresentaram piora no desenvolvimento motor e na autonomia em comparação a crianças saudáveis, embora a severidade da doença não tenha relação direta com a qualidade de vida.

Podemos dizer que a qualidade de vida envolve a adaptação, o ajustamento psicológico e social, como também a forma como a criança é acompanhada, seja na escola, nas instituições de saúde ou em outras ocasiões, além da forma de se expressar e se constituir como sujeito.

Ciente da complexidade que caracteriza a cardiopatia, autores (Kuczynski, Assumpção Júnior, 2010) afirmam que as crianças são reflexo do macro e do microambiente em que estão inseridas e, dependendo do contexto, pode haver influência a longo prazo no desenvolvimento social e psicológico.

Nesta perspectiva, um estudo (Martins, Juvenal, Teófilo, Cunha, 2004) relatou que avaliar as condições ambientais e os estímulos que são oferecidos às crianças e suas famílias pode fornecer dados importantes para o incremento de intervenções preventivas e promocionais em saúde; a motivação e atitudes das crianças são fatores importantes de adaptação positiva, os quais proporcionam uma melhor qualidade de vida do que a complexidade da condição médica (Majnemer, Limperopoulos, Shevell, Rosenblatt, Tchervenkov, 2006).

Silva e col. (2006) em um estudo no qual abordaram um grupo de adolescentes cardiopatas concluíram que o suporte social aumenta a resiliência e promove melhor adaptação à doença.

Com isso em mente, Williams e col. (2002) e Chen e col. (2007) afirmam que os jovens passam por um processo de desafiar a autoridade dos pais em face da superproteção deles quando estabelecem uma linha tênue entre os comportamentos saudáveis - as práticas alimentares e os exercícios físicos - e os de risco -uso de drogas e práticas sexuais de risco-, os quais irão se manifestar pela primeira vez.

Levando em consideração esse contexto, o sociólogo norte-americano Aaron Antonovsky (1979) cunhou o termo senso de coerência (SOC) que reflete a forma como o indivíduo percebe a sua saúde e enfrenta os agentes estressores; o estudioso propõe um tripé para descrever o termo: compreensão - o quanto os estímulos internos e externos recebidos durante a infância são estruturados, previsíveis e explicáveis -, manejo - percepção dos recursos disponíveis para lidar com situações estressoras - e significado - capacidade do indivíduo de acreditar que sua vida tem sentido, encontrar motivação e assumir o controle sobre ela ${ }^{31}$.

Nos estudos de Luyckx e col. (2012) e Moons e col. (2002) ao observarem grupos de adolescentes cardiopatas com forte senso de identidade, concluíram que esses sujeitos se mostram mais satisfeitos com suas vidas, possuindo um sentimento dinâmico e duradouro de confiança em si e em seu ambiente.

b) Desenvolvimento infantil

O desenvolvimento infantil é influenciado não só por variáveis múltiplas e pela natureza biológica, mas também por fatores sociais e culturais, os quais podem ser decisórios na modificação de respostas nas diversas fases do desenvolvimento neuropsicológico (Mello, Miranda, Muszkat, 2004). 
Pilz e Schumann (2007) observaram algumas variáveis que podem interferir no desenvolvimento infantil: escolaridade da mãe, renda, problemas na gestação, intervalos entre as gestações, condições do bebê ao nascer, presença de alguma patologia crônica, participação e presença paterna, saúde mental dos pais, ambiente hostil e estimulação oferecida a criança.

Segundo o estudo de Uzark e col. (1998) as crianças com cardiopatia congênita podem apresentar atraso no desenvolvimento, entretanto, esse atraso ocorrerá de acordo com o estágio em que a criança se encontra, ou seja, se a criança já realizou cirurgia para correção de má formação cardíaca, se está no aguardo da cirurgia ou se está em acompanhamento clínico. Além disso, um estudo (McGrath Em Wypij, Rappaport, Newburger, Bellinger, 2004) aponta que uma criança de um ano aparentemente normal em seu desenvolvimento e que passou por cirurgia cardíaca na infância pode apresentar alterações no neurodesenvolvimento aos 4 ou 8 anos de idade.

Silva (2006) destaca que a presença de alguma patologia crônica, como é o caso da cardiopatia congênita, altera o desenvolvimento físico, motor, cognitivo e neurológico; consequentemente, a criança cardiopata pode ter problemas comportamentais, atrasos na fala, falta de atenção ou hiperatividade, alterações na autoestima e na autoimagem e dificuldades acadêmicas (Sable, Foster, Uzark, Bjornsen, Canobbio, Connolly, et al.2011).

Como vimos acima, os autores afirmam em sua maioria que crianças e adolescentes com cardiopatia congênita possuem alterações tanto fisiológicas quanto neuropsicológicas e isso influencia no modo como se relacionam consigo mesmas e com os outros, sendo imprescindível propor práticas interdisciplinares relacionadas a saúde visando a inclusão e o tratamento coerente com cada indivíduo, levando em consideração as suas particularidades.

\section{Método}

Trata-se de um estudo descritivo, exploratório e longitudinal. A natureza da pesquisa é quali-quanti pois a pesquisa qualitativa busca identificar os efeitos de um determinado fenômeno a ser estudado, e a quantitativa busca a causa desses efeitos. É o que este trabalho pretende mostrar ao trazer os resultados e sua análise.

Local: ambulatório de cardiopediatria do Hospital do Coração -HCor - do município de São Paulo.

Sujeitos: crianças entre 10 meses e 09 anos com cardiopatia congênita e suas mães.

\section{Critérios de inclusão:}

Serem atendidas regularmente no ambulatório de cardiopediatria, que se dispuseram a comparecer semanalmente para os atendimentos propostos e assinaram o termo de consentimento livre e esclarecido.

\subsection{Procedimento}

As crianças e suas famílias foram convocadas a participar da pesquisa que oferecia acompanhamento terapêutico semanal multidisciplinar formado por fonoaudiólogas, fisioterapeutas, psicólogas e psicopedagoga e pela equipe médica conforme necessidade de cada caso.

\section{Com as crianças:}

Etapa 1: Observação

1) As crianças foram observadas, pela equipe multidisciplinar na sala de espera para o atendimento médico;

2) Durante a observação, as terapeutas interagiam com as crianças;

3) A partir desta observação, definiu-se a avaliação e os atendimentos a serem propostos para cada criança, a saber: 1) atendimento em grupo; 2) atendimento individual; 3 ) atendimento individual e em grupo; 
Etapa 2: Avaliação e intervenção

1) Entrevistas com os familiares e/ou cuidadores em que foram coletados os dados cirúrgicos, nosológicos e o histórico do desenvolvimento neuropsicomotor e de linguagem das crianças e os dados do perfil sociodemográfico das mães;

2) Avaliação das crianças indicadas para o atendimento individual pelo Protocolo de Observação Comportamental (PROC) e pelos Marcos do desenvolvimento dos Centros de Controle e Prevenção de Doenças (CDC) dos Estados Unidos;

3) Atendimento terapêutico individual e em grupo;

4) Reavaliação do PROC com intervalo de três a seis meses ao longo de um ano.

Etapa 3: Análise dos dados

1) Os dados coletados foram registrados e descritos de acordo com as variáveis analisadas;

2) Aos dados foi aplicada análise estatística simples;

3) Foram feitas correlações entre os achados da pesquisa e a literatura de referência.

\section{Com as mães}

Etapa 1: Entrevista:

1) Aplicação do questionário WHO-QOL para levantar dados sobre a qualidade de vida das famílias envolvidas

2) Aplicação do questionário sobre Estresse Parental para conhecer os efeitos das várias internações Etapa 2: Intervenção:

1) Para as famílias foi proposto a participação em um grupo terapêutico de pais.

Ética: A pesquisa tem a concordância de responsáveis legais e assinatura de termo livre esclarecido ( ${ }^{\circ}$ do CAAE: 02000718.7.3001.5482).

\section{Resultados}

\section{Das crianças}

Levantamos a idade e a escolaridade das 21 crianças, que podem ser observadas no Quadro 1. Observamos que duas delas não frequentam a escola e, embora seja um número pequeno (menos de 10\%), identificamos que o que motiva essa decisão dos pais são seus temores de que seus filhos possam se machucar durante as atividades recreativas ou de educação física. Cabe ressaltar que tanto a orientação médica quanto a fisioterapêutica é de que as crianças participem dessas atividades pois não há risco de danos a sua saúde. Mesmo as mães cujos filhos frequentam a escola, não o fazem sem preocupação. 
Quadro 1. Idade e escolaridade das crianças.

\begin{tabular}{|l|l|l|}
\hline Sujeitos & Idade & Escolaridade \\
\hline S1 & 7 & Ensino fundamental I \\
\hline S2 & 8 & Ensino fundamental I \\
\hline S3 & 9 & Ensino fundamental I \\
\hline S4 & 5 & Pré 2 \\
\hline S5 & 5 & Não frequenta escola \\
\hline S6 & 6 & Ensino fundamental I \\
\hline S7 & 10 & Ensino fundamental I \\
\hline S8 & 6 & Ensino fundamental I \\
\hline S9 & 9 & Ensino fundamental I \\
\hline S10 & 5 & Pré 2 \\
\hline S11 & 4 & Pré 1 \\
\hline S12 & 10 & Ensino fundamental II \\
\hline S13 & 7 & Ensino fundamental I \\
\hline S14 & 7 & Não frequenta escola \\
\hline S15 & 6 & Ensino fundamental I \\
\hline S16 & 5 & Pré 2 \\
\hline S17 & 3 & Não frequenta escola \\
\hline S18 & 11 & Ensino fundamental II \\
\hline S19 & 10 & Ensino fundamental II \\
\hline S20 & 9 & Ensino fundamental I \\
\hline S21 & 7 & Ensino fundamental I \\
\hline
\end{tabular}

Fonte: Autores.

De forma geral, as crianças acompanham a escolaridade prevista, ou seja, estão cursando o Fundamental I entre as idades de 6 a 10 anos, conforme preconizado pelo Ministério da Educação. Temos inclusive três crianças, de 10 e 11 anos, que já estão cursando o Fundamental II.

No quadro a seguir, identificamos o tempo de hospitalização e internação das crianças, em número de dias, para a realização das cirurgias exigidas pelo quadro de cardiopatia congênita. 
Quadro 2. Sujeitos, número de cirurgias e tempo de internação.

\begin{tabular}{|l|c|l|}
\hline Sujeitos & Número de cirurgias & Dias de internação \\
\hline S1 & 3 & 51 \\
\hline S2 & 3 & 102 \\
\hline S3 & 2 & 98 \\
\hline S4 & 2 & 83 \\
\hline S5 & 2 & 37 \\
\hline S6 & 3 & 126 \\
\hline S7 & Sem informação & - \\
\hline S8 & 1 & 16 \\
\hline S9 & 2 & 25 \\
\hline S10 & 3 & 79 \\
\hline S11 & Sem informação & - \\
\hline S12 & 3 & 387 \\
\hline S13 & 3 & 209 \\
\hline S14 & 3 & 62 \\
\hline S15 & 3 & 39 \\
\hline S16 & 2 & 64 \\
\hline S17 & 1 & 174 \\
\hline S18 & 3 & 147 \\
\hline S19 & 3 & 283 \\
\hline S20 & 3 & 57 \\
\hline S21 & 2 & 21 \\
\hline Total & & 2.060 \\
\hline
\end{tabular}

Fonte: Autores.

Observa-se que o maior tempo de internação foi o de S12 que ficou internado por 387 dias, enquanto o menor tempo de internação foi o de S15 que permaneceu internado por 39 dias. O número de cirurgias por que passaram as crianças variou de 1 a 3 , sendo que mais de $50 \%$ dos sujeitos passaram pelas 3 cirurgias.

Constata-se, também, que os sujeitos que têm maior número de dias de internação, fizeram as 3 cirurgias - S2, S6, S12, S13, S18 e S19 - com exceção do S17 que tem um número elevado de dias de internação e fez apenas 1 cirurgia.

\section{Avaliação de linguagem - PROC:}

Feito o levantamento geral da demanda da população alvo, identificamos que algumas crianças apresentavam alguma queixa relativa a fala, trazida, em geral, por suas mães e, também, observada pelos profissionais na sala de espera.

Assim, da amostra de 21 crianças, seis foram avaliadas pelo Protocolo de Observação Comportamental (PROC) por serem aquelas que apresentavam queixas de alterações de fala. O PROC ${ }^{4}$ é um Protocolo de observação Comportamental para crianças de 12 a 48 meses realizado em sessões individuais de 30 a 40 minutos. Este protocolo proporciona a descrição de variáveis qualitativas e quantitativas, indicando que a pontuação máxima do teste é de 70 pontos para habilidades comunicativas; 60 pontos para compreensão da linguagem oral; 70 pontos para aspectos do desenvolvimento cognitivo e 200 pontos no escore total. O protocolo avalia três áreas: 1. Habilidades Comunicativas (1.a - habilidades dialógicas, 1.b - funções comunicativas, 1.c - meios de comunicação e 1.d - níveis de contextualização da linguagem), 2. Compreensão Verbal e 3. Aspectos do Desenvolvimento Cognitivo (3.a - formas de manipulações dos objetos, 3. b - nível de desenvolvimento do simbolismo,3.c - nível de organização do brinquedo e 3.d - imitação).

Sua escolha se deu por ser um protocolo de rápida aplicação e por permitir a observação do comportamento geral da criança em contexto semiestruturado.

\footnotetext{
${ }^{4}$ Hage SRV, Pereira TC, Zorzi, J Protocolo de Observação Comportamental - PROC: valores de referência para uma análise quantitativa, Rev. Cefac
} 
As crianças cujas queixas de problemas de linguagem foram confirmadas pelo teste, foram encaminhadas para atendimento grupal além do atendimento individual, pois entendeu-se que o grupo poderia se beneficiar do tratamento. O Quadro 3 abaixo traz os resultados do PROC por área de avaliação e após um tempo de 3 meses a um ano de intervenção nas seis crianças encaminhadas para avaliação

Quadro 3. Protocolo de Observação Comportamental (PROC) e atendimento sugerido de acordo com a pontuação atingida na avaliação inicial.

\begin{tabular}{|c|c|c|c|c|c|c|}
\hline Sujeitos & Idade & $\begin{array}{l}\text { Tipo de } \\
\text { atendimento }\end{array}$ & $\begin{array}{l}\text { Habilidades } \\
\text { Comunicativas }\end{array}$ & Compreensão & Cognitivo & $\begin{array}{l}\text { Pontuação } \\
\text { final* }\end{array}$ \\
\hline S5 & 54 meses & $\begin{array}{l}\text { Individual e } \\
\text { grupo }\end{array}$ & 66 & 50 & 43 & 159 \\
\hline S8 & 53 meses & $\begin{array}{l}\text { Individual } \\
\text { grupo }\end{array}$ & 52 & 50 & 54 & 166 \\
\hline S10 & 43 meses & $\begin{array}{l}\text { Individual } \mathrm{e} \\
\text { grupo }\end{array}$ & 17 & 40 & 8 & 65 \\
\hline S11 & 53 meses & Nenhum & 52 & 60 & 39 & 141 \\
\hline $\mathrm{S} 16$ & 41 meses & $\begin{array}{l}\text { Individual e } \\
\text { grupo }\end{array}$ & 17 & 40 & 12 & 23 \\
\hline S17 & 17 meses & $\begin{array}{l}\text { Individual } \\
\text { grupo }\end{array}$ & 10 & 20 & 3 & 33 \\
\hline
\end{tabular}

Fonte: Autores.

Como pode-se observar no Quadro 4, destas, 3 crianças (S10, S16 e S17) eram não verbais e apresentavam dificuldades em: intenção comunicativa, compreensão de linguagem oral, imitação sonora e imitação gestual, estando na fase inicial do desenvolvimento sensório motor.

As outras três crianças (S5, S8 e S11) apresentavam comunicação intencional plurifuncional, ampla participação em atividade dialógica por meios verbais ligados ao contexto imediato, compreensão de ordens com até duas ações ligados ao contexto, estando na fase representativa do desenvolvimento cognitivo.

Quadro 4. Protocolo de Observação Comportamental (PROC) - Segunda avaliação após atendimento fonoaudiológico.

\begin{tabular}{|l|c|c|c|c|c|}
\hline Sujeitos & Tempo de atendimento & Habilidades Comunicativas & Compreensão & Cognitivo & Pontuação final* \\
\hline S5 & 03 meses & 70 & 50 & 52 & 172 \\
\hline S8 & Não realizado & - & - & - & - \\
\hline S10 & 07 meses & 25 & 50 & 37 & 112 \\
\hline S11 & Não realizado & - & - & & - \\
\hline S16 & 05 meses & 31 & 40 & 23 & 94 \\
\hline S17 & 05 meses & 19 & 50 & 7 & 76 \\
\hline
\end{tabular}

*MÁXIMO = 200 PONTOS. Fonte: Autores.

O Quadro 5 compara os resultados obtidos na primeira e na segunda avaliação, indicando ganhos importantes no campo da linguagem com a intervenção. 
Quadro 5. Resultados, em pontos, das avaliações segundo o PROC.

\begin{tabular}{|c|c|c|c|}
\hline \multicolumn{1}{|l|}{ Sujeitos } & Primeira avaliação & Segunda avaliação & Ganho em pontos \\
\hline S5 & 159 & 172 & 13 \\
\hline S8 & 166 & - & - \\
\hline S10 & 62 & 112 & 50 \\
\hline S11 & 141 & - & - \\
\hline S16 & 23 & 94 & 71 \\
\hline S17 & 33 & 78 & 45 \\
\hline
\end{tabular}

*MÁXIMO = 200 PONTOS. Fonte: Autores

\section{Avaliação dos marcos do desenvolvimento - LTSAE5:}

O Learn The Signs Act Early é uma lista de acompanhamento dos marcos do desenvolvimento divulgada pelo CDC (Centers for Disease Control and Prevention's dos Estados Unidos $\left.{ }^{6}\right)$. Os marcos do desenvolvimento são apresentados em fichas divididas por idades: 2 meses; 4 meses; 6 meses; 9 meses; 1 ano; 18 meses; 2 anos; 3 anos; 4 anos e 5 anos.

Preencheu-se as fichas do LTSAE como forma complementar de levantamento de dados sobre o desenvolvimento geral em 7 crianças. São fichas nas quais a mãe ou cuidadora observa e assinala a presença de comportamentos em 4 áreas: social/emocional; linguagem/comunicação; cognitivo e movimento/desenvolvimento físico e foram usadas para delinear o perfil de desenvolvimento das crianças. Podemos observar os resultados no Quadro 6.

Quadro 6. Resultados CDC.

\begin{tabular}{|l|c|l|}
\hline Sujeitos & Idade cronológica & LTSAE \\
\hline S5 & 5 anos & 3 anos \\
\hline S8 & 5 anos & 4 anos \\
\hline S10 & 5 anos & 2 anos \\
\hline S13 & 7 anos & 3 anos \\
\hline S14 & 7 anos & 4 anos \\
\hline S16 & 5 anos & $1 ; 06$ anos \\
\hline S17 & 3 anos & 1 ano \\
\hline
\end{tabular}

Fonte: Autores.

Foi possível observar que as 7 crianças que passaram pelo LTSAE apresentaram alterações nas áreas avaliadas pelos marcos do desenvolvimento - social/emocional, linguagem/comunicação, cognitivo e movimento /desenvolvimento físico.

Os resultados, quando comparados aos esperados para as respectivas idades cronológicas, pontuaram $50 \%$ abaixo e correspondem a resultados compatíveis (entre 75 e 100\% de presença) com o desenvolvimento de crianças com 1 ano a menos.

Quando a alteração de linguagem presente dizia respeito a condições do dizer da criança (3 crianças- S10, S16 e S17), ou seja, a dificuldades relacionadas a construção de um lugar de falante, os marcos do desenvolvimento correspondiam a uma idade cronológica defasada entre 2 e 2;6 anos. Ou seja, quanto mais nova a criança, mais evidentes e significativas são as alterações no desenvolvimento; com a maturação e, consequentemente, com a emergência da linguagem, as diferenças vão se atenuando e ficam menos expressivas.

\footnotetext{
${ }^{5}$ Learn the signs act early - aplicativo desenvolvido pelo CDC- Centers for Disease Control and Preventions

${ }^{6}$ Adapted from Caring for Your Baby and Young Child: Birth to Age 5, Fifth Edition, edited by Steven Shelov and Tanya Remer Altmann (C) 1991, 1993, 1998, 2004, 2009 by the American Academy of Pediatrics and Bright Futures: Guidelines for Health Supervision of Infants, Children, and Adolescents, Third Edition, edited by Joseph Hagan, Jr., Judith S. Shaw, and Paula M. Duncan, 2008, Elk Grove Village, IL: American Academy of Pediatrics. This milestone checklist is not a substitute for a standardized, validated developmental screening tool.
} 


\section{Das mães:}

Quando o projeto teve início, em fevereiro de 2018, tínhamos um total de 26 crianças e suas mães. Este número foi a base para a análise dos questionários aplicados com as mães.

Foram aplicados os questionários Parental Stress Scale: Neonatal Intensive Care Unit (PSS: NICU (2012) ${ }^{7}$ e o Questionário de Qualidade de Vida (WHOQOL-BREF (1994) ${ }^{8}$ em 26 mães.

As respostas ao WHOQOL-BREF indicaram que 17\% das mães reconhecem a sua qualidade de vida como boa; $21 \%$ como precisa melhorar e 60\% como regular. A escala de estresse na UTI que avalia três áreas - 1) sons e imagens, 2) aparência e o comportamento do bebê e 3) alteração no papel de mãe/pai - apontou que a aparência e o comportamento do bebê ou da criança é uma área que causa grande estresse no período de internação, com $84 \%$ das respostas. A área 3 (alteração no papel de mãe/pai) obteve $100 \%$ das respostas em nível máximo (nível 5) como fator causador de estresse parental. Lembramos que, nos casos de cardiopatia congênita, as crianças passam por duas a três cirurgias para as quais são hospitalizadas em UT infantil e em quarto hospital.

No momento do levantamento do histórico das famílias, o número de sujeitos havia diminuído para 21 pois, algumas mães, por razões pessoais, decidiram não dar continuidade a sua participação na pesquisa.

Os primeiros resultados extraídos desse histórico são os de idade e escolaridade das mães. Não obtivemos os dados de quatro das 21 mães. O Quadro 7 resume os resultados referentes a 17 mães.

Quadro 7. Idade e escolaridade das mães.

\begin{tabular}{|l|l|l|}
\hline Sujeitos & Idade & \multicolumn{1}{|c|}{ Nível de escolaridade } \\
\hline S1 & 38 & Ensino médio completo \\
\hline S2 & 30 & Ensino médio completo \\
\hline S3 & 39 & Ensino médio completo \\
\hline S4 & 33 & Ensino médio completo \\
\hline S5 & 36 & Ensino superior completo \\
\hline S6 & Sem dados & - \\
\hline S7 & 50 & Ensino médio completo \\
\hline S8 & 35 & Ensino médio completo \\
\hline S9 & 39 & Ensino médio completo \\
\hline S10 & 30 & Ensino fundamental completo \\
\hline S11 & Sem dados & - \\
\hline S12 & Sem dados & \\
\hline S13 & 32 & Ensino médio completo \\
\hline S14 & 38 & Ensino superior incompleto \\
\hline S15 & 38 & Ensino superior completo \\
\hline S16 & 42 & Ensino fundamental incompleto \\
\hline S17 & 29 & Ensino médio completo \\
\hline S18 & Sem dados & \\
\hline S19 & 35 & Ensino médio completo \\
\hline S20 & 41 & Ensino médio completo \\
\hline S21 & 39 & Ensino fundamental completo \\
\hline & & \\
\hline
\end{tabular}

Fonte: Autores.

A média de idade das mães é de 37 anos embora varie em uma faixa que vai de 29 a 50 anos. Do ponto de vista da escolaridade, $82,35 \%$ têm de ensino médio completo a ensino superior, completo ou incompleto.

\footnotetext{
${ }^{7}$ Souza Sandra Regina de, Dupas Giselle, Balieiro Maria Magda Ferreira Gomes. Adaptação cultural e validação para a língua portuguesa da Parental Stress Scale: Neonatal Intensive Care Unit (PSS:NICU). Acta paul. enferm. [Internet]. 2012 [cited 2021 May 05]; 25(2): 171-176. Available from: http://www.scielo.br/scielo.php?script=sci_arttext\&pid=S0103-21002012000200003\&lng=en. https://doi.org/10.1590/S0103-21002012000200003.

${ }^{8}$ The Whoqol Group. The development of the World Health Organization quality of life assessment instrument (the WHOQOL). In: Orley J, Kuyken W, editors. Quality of life assessment: international perspectives. Heidelberg: Springer Verlag;1994. p 41-60.
} 


\section{O grupo de pais}

Os encontros iniciaram em maio e terminaram em dezembro de 2019. Foram realizados 18 encontros de uma hora cada. Todos os encontros foram gravados integralmente sob a permissão de todos os participantes. A previsão era de ocorrer um encontro por semana, todas as segundas-feiras, das $10 \mathrm{~h}$ às $11 \mathrm{~h}$, com recesso no mês de julho decorrente das férias escolares. Não ocorreram 4 encontros por razões diversas. Antes de cada encontro, o coordenador convidava os pais, mães, parentes e responsáveis pelas crianças, que estavam na sala de espera, para participarem do grupo. Ninguém era obrigado a participar. Participaram dos 18 encontros um total de 23 pessoas, entre mães, pais e cuidadores. A participação masculina, seja por pais ou por demais parentes, foi bastante reduzida, não só com relação aos encontros, mas também ao comparecimento ao HCor para participação das demais atividades. A média de participantes por encontro foi de 4 pessoas.

Os encontros entre os pais e o psicanalista foi mediado pelo método da Conversação, dispositivo psicanalítico lacaniano proposto por Jacques-Alain Miller (2005) e desenvolvido como metodologia de pesquisa-intervenção por Santiago (2011). Este dispositivo consiste em deixar o outro falar, sem direcionamentos temáticos e, por meio de cortes em dados significantes, motivar o participante a falar mais sobre o assunto e, assim, gerar diversas cadeias significantes, ou descolar o sujeito de uma dada posição subjetiva/discursiva ou, ainda, para provocar a fala de alguém. Os temas que mais se tornaram presentes nos encontros indicam quão simbiótica é a relação dos pais com essas crianças, e os possíveis prejuízos que podem decorrer da qualidade dessa relação. O excesso de proteção ao filho foi entendido como autoproteção. Pais que largaram seus trabalhos, seus estudos, para cuidarem integralmente de seus filhos. Tal excesso ocorre na tentativa de evitar o inevitável, a morte. Em razão do pouco tempo de vida, é preciso viver em excesso. É preciso viver mãe e filho, pai e filho, em uma bolha. Bolha que precisará ser rompida para que a criança possa vir a ser o autor da sua própria vida, dos seus próprios dizeres e quereres, ainda que para isso tenha que correr riscos. Caso contrário, perderá a capacidade de tomar qualquer decisão, de construir qualquer caminho... faltará à criança o desejo de viver e de fazer qualquer coisa, pois seu desejo, estando alienado ao desejo do outro, a impedirá de arriscar-se e, assim, de não conseguir fazer ou ser nada na vida (Freire \& Ferraz, 2021)

\section{Discussão}

Não se observou correlação de qualquer natureza entre tempo de internação e desenvolvimento posterior da criança. No entanto, várias mães relataram que após a segunda cirurgia, momento de grande desenvolvimento da criança (por volta dos 5;6 meses), houve um retrocesso que foi superado nos meses seguintes e, aparentemente, não deixou marcas definitivas (Uzark, Lincoln, Lamberti, Mainwaring, Spicer, Moore, 1998).

A correlação tempo de internação x resultados dos Testes PROC e CDC não apresentaram significância, ou seja, as 07 crianças analisadas não apresentaram resultados discrepantes quando comparadas entre si ou entre si e o tempo maior ou menor de internação.

Também não se encontrou correlação entre o desenvolvimento ao longo do ano e o tempo de internação ou o número de cirurgias.

Observa-se que todas as crianças têm uma condição adversa na linguagem e que, dentre estas manifestações, há uma grande variedade de perfis que vão desde crianças que não se comunicam pela via da palavra até crianças que falam, mas apresentam dificuldade na narratividade de seu discurso. Tal variabilidade de manifestações nos leva a afirmar que não há relação direta entre aspectos da linguagem que estariam alterados e a cardiopatia congênita. Por outro lado, a presença de alguma manifestação na linguagem em todas as crianças avaliadas, nos direciona a inferir que crianças com cardiopatia congênita estão expostas a uma condição que interfere no desenvolvimento da linguagem, ou no plano das condições do seu dizer (McGrath, Wypij, Rappaport, Newburger, Bellinger, 2004; Sable, Foster, Uzark, Bjornsen, Canobbio, Connolly, et al., 2011). Ainda que não se possa quantificar, por meio de dados objetivos, o desenvolvimento do grupo de 18 crianças, pode-se 
afirmar que a maioria avançou desde onde estava quando avaliada, após a intervenção proposta, sustentando a importância de um tratamento multidisciplinar (Freud,1972; Portaria $n^{\circ} 1727,2017$ ).

Outro dado obtido é sobre a escolaridade das crianças cardiopatas que, considerando um intervalo possível de idade para cursar os respectivos anos escolares, estão dentro do esperado, o que parece confirmar o fato de que nem todas as crianças cardiopatas irão apresentar atraso no desenvolvimento com consequências para a nível de escolaridade (Uzark, Lincoln, Lamberti, Mainwaring, Spicer, Moore,1998).

Em relação as idades e escolaridades das mães, os dados obtidos nos ajudam a traçar um perfil das mães deste grupo a maior parte delas conta com o ensino médio completo e tem, em média, 37 anos. Além disso, por meio de relatos no grupo de pais, constata-se quase cem por cento das mães é a principal acompanhante da criança, o que está sincronizado com dados da literatura. Fato este que pode ser considerado benéfico para a criança cardiopata que, segundo dados da literatura, se beneficia com a presença das mães durante seu tratamento (Jansen, Silva, da Novello, Guimarães, Silva da,2000; Gomes, Erdmann, 2005; Molina, Fonseca, Waidman, Marcon, 2009).

Outro dado evidente nos relatos, é o fato das mães terem dificuldade de sustentar uma vida profissional junto com a demanda de cuidado dos filhos - a maior parte delas, abdicou do trabalho remunerado, fora de casa, dedicando-se, exclusivamente, aos cuidados dos filhos que, pela rotina de médicos, tratamentos e cirurgia, demandam tempo e atenção (Ribeiro, 2002).

\section{Conclusão}

O presente trabalho foi promissor na medida em que nos aproximou da compreensão do impacto dos possíveis fatores de risco para o desenvolvimento na criança cardiopata.

Ainda que não possamos mostrar por meio de dados objetivos sobre a evolução no desenvolvimento psicomotor e de linguagem pela aplicação dos protocolos descritos no estudo, pode-se afirmar que a maioria avançou desde onde estava quando avaliada pela primeira vez, após a intervenção proposta.

De acordo com dados do grupo de mães e da própria equipe, as mães se mostraram mais apropriadas e seguras em relação ao desenvolvimento de seus filhos e mais ativas no processo terapêutico.

Constatou-se, também, que as crianças ficaram mais tranquilas e receptivas para a consulta médica ao longo dos atendimentos realizados, principalmente das atividades lúdicas realizadas na sala de espera; e a equipe médica passou a estar mais atenta a questões subjetivas das crianças.

É preciso ressaltar que o estudo apresenta limitações devido ao fato de ter sido realizado em apenas um ambulatório, com um número reduzido de participantes, durante um curto período. Sugere-se conduzir outros mapeamentos e estudos neste contexto de forma a ampliar a compreensão acerca dos fatores de risco para o desenvolvimento neuropsicomotor e de linguagem em crianças com cardiopatia congênita, bem como observar os efeitos da intervenção multidisciplinar no desenvolvimento destas crianças. Para os trabalhos futuros, sugerimos que o uso de um teste padrão ouro, como o Denver, levaria os resultados para um patamar universal, permitindo a comparação dos achados com os da literatura nacional e internacional. Um número maior de crianças também daria maior sustentabilidade para os resultados.

\section{Referências}

Andropoulos D. B., Hunter J. V., Nelson D. P., Stayer S. A., Stark A. R., McKenzie E. D., et al. (2010). Brain immaturity is associated with brain injury before and after neonatal cardiac surgery with high-flow bypass and cerebral oxygenation monitoring. J Thorac Cardiovasc Surg. 139(3), 543-556.

Antonovsky A. (1979). Health, stress, and coping. Jossey-Bass.

Aita K. M. S. C. \& De Souza A. M. (2016). Cenas sobre a morte, reveladas pela criança abrir o coração. Rev NUFFEN;8(1):141-162. 
Barreto T. S. M., Sakamoto V. T. M., Magagnin J. S., Coelho D. F., Waterkemper R. \& Canabarro S. T. (2016) Vivência de pais de crianças com cardiopatia congênita: sentimentos e obstáculos. Rev. Rene. 17(1): 128-36.

Beca J., Gunn J. K., Coleman L., Hope A., Reed P. W., Hunt R. W., et al. (2013) New white matter brain injury after infant heart surgery is associated with diagnostic group and the use of circulatory arrest. Circulation.127 (9): 971-979.

Bertoletti J., Marx G. C., Júnior Hattge S. P. \& Pellanda L. C. (2014) Qualidade de vida e cardiopatia congênita na infância e adolescência. Arq. Bras. Cardiol. 102(2): 192-198.

Chen C. W., Chen Y. C. Chen M. Y., Wang J. K., Su W. J. \& Wang H. L. (2007). Health-promoting behavior of adolescents with congenital heart disease. J Adolesc Health. 41(6): 602-9.

Cunha M. C., Silva M. F. F. \& Palladino R. R. R. (2014). Hospitalizações precoces e problemas de linguagem oral: coocorrências em crianças. Rev. Dist. Comum. 23(3).

Dos Santos S. R., Dias I. M. A. V., Salimena A. M. de O \& Bara V. M. F. (2011). A vivência dos pais de uma criança com malformações congênitas. Rev. Min. Enferm. 15(4): 491-497.

Freire, R. M. F \& Ferraz, M. A. M. (2021) - A criança para além da cardiopatia congênita pelo olhar da análise de discurso in: Dalexon Sérgio da Silva; Claudemir dos Santos Silva (Orgs.) Pêcheux em (dis) curso: entre o já-dito e o novo. Uma homenagem à professora Nadia Azevedo. 2, Pedro e João editores, p. 461

Freud S. (1972). Fragmento da análise de um caso de histeria: [o caso Dora]. Imago.

Gaynor J.W., Stopp C., Wypij D., Andropoulos D. B., Atallah J., Atz A. M., et al. (2015). Neurodevelopmental outcomes after cardiac surgery in infancy. Pediatrics. 135(5): 816-825.

Gomes G. C. \& Erdmann A. L. (2005). O cuidado compartilhado entre a familia e a enfermagem à criança no hospital: uma perspectiva para a sua humanização. Ver Gaúcha Enferm. 26(1): 20-30.

Hövels-Gürich H. H. (2016). Factors infuencing neurodevelopment after cardiac urgery during infancy. Front Pediatr. 137(4).

Hövels-Gürich H. H. (2012). Psychomotor development of children with congenital heart defects. Causes, prevalence and prevention of developmental disorders after cardiac surgery in childhood. Monatsschr Kinderheilkd.; 216(3): 132-140.

Jansen D., Silva K. V. P. T. da Novello R., Guimarães T. C. \& Silva V. G. da. (2000). Assistência de enfermagem à criança portadora de cardiopatia. Rev. SOCERJ. 13(1): 22-29.

Jerusalinsky A. (2004). Psicanálise e desenvolvimento infantil: um enfoque transdisciplinar. Artes e Ofícios.

Kirschbaum, C. (2013). Decisões entre pesquisas quali e quanti sob a perspectiva de mecanismos causais. Revista Brasileira de Ciencias Sociais, 28(82).

Krol Y., Grootenhuis M. A., Destrée-Vonk A., Lubbers L. J., Koopman H. M. \& Last B. F. (2003). Health related quality of life in children with congenital heart disease. Psychol \& Health.18(2): 251-260.

Kuczynski E.\& Assumpção Júnior F. (2010). Qualidade de vida na infância e na adolescência: orientações para pediatras e profissionais da saúde mental. Artmed.

Leal L. S., Silva da R. L. M., Aita K. M. S. C., Monteiro R. P. de A. \& de Montalvão T. C. (2016). Avaliação do desenvolvimento motor de crianças portadoras de cardiopatia congênita. International Journal of Cardiovascular Sciences. 29(2):103-109.

Licht D. J., Shera D. M., Clancy R. R., et al. (2009) Brain maturation is delayed in infants with complex congenital heart defects. J Thorac Cardiovasc Surg.; 137(3): 529-536

Licht D. J., Wang J., Silvestre D. W., Nicolson S. C., Montenegro L. M., Wernovsky G., et al. (2004). Preoperative cerebral blood flow is diminished in neonates with severe congenital heart defects. J Thorac Cardiovasc Surg.; 128(6): 841-849.

Luyckx K., Missotten L. C., Goossens E. \& Moons P. (2012). Individual and contextual determinants of quality of life in adolescents with congenital heart disease. J Adolesc Health. 51(2): 122-8.

Majnemer A., Limperopoulos C., Shevell M., Rosenblatt B., \& Tchervenkov C. (2006) Health and well-being of children with congenital cardiac malformations, and their families, following open-heart surgery. Cardiol Young.

16(2): 157-64

Mari M. A., Cascudo M. M. \& Alchieri J. C. (2016). Congenital heart disease and impacts on child development. Braz. J. Cardiovasc. Surg. 31 (1): 31-7.

Mari M. A. \& Alchieri J. C. (2012). Desenvolvimento neuropsicológico infantil e variáveis biopsicossociais em crianças com cardiopatias congênitas: identificação de marcadores psicológicos. FIEP BULLETIN - Special Edition - ARTICLE II. p. 82.

Marino B. S., Lipkin P. H., Newburger J. W., Peacock G., Gerdes M., Gaynor J. W., et al. (2012). Neurodevelopmental outcomes in children with congenital heart disease: evaluation and management. A Scientific statement from the American Heart Association. Circulation. 126(9): 1143-1172.

Martins M. F. D., Juvenal S. D. S., Teófilo E. \& Cunha M. D. C. (2004) Qualidade do ambiente e fatores associados: um estudo em crianças de Pelotas, Rio Grande do Sul, Brasil. Caderno de Saúde Pública. 20(3): 710-718. 
Research, Society and Development, v. 10, n. 11, e83101119538, 2021

(CC BY 4.0) | ISSN 2525-3409 | DOI: http://dx.doi.org/10.33448/rsd-v10i11.19538

McGrath E., Wypij D., Rappaport L. A., Newburger J. W. \& Bellinger D. C. (2004). Prediction of IQ and achievement at age 8 years from neurodevelopmental status at age 1 year in children with D-transposition of the great arteries. Pediatrics.114(5): 572-6.

Mello C. B., Miranda M. C. \& Muszkat M. (2004). Neuropsicologia do desenvolvimento. In: Andrade V., Santos F., \& Bueno O. F. A., organizadores. Neuropsicologia Hoje. Artes Médicas; p. 211-224.

Miller J.A. (2005). Efectos terapéuticos rápidos. Paidós;

Ministério da Saúde. Gabinete do Ministro. Portaria no . 1727, de 11 de julho de 2017. Brasília, 2017.

Molina R. C. M., Fonseca E. L., Waidman M. A. P. \& Marcon S. S. (2009) A percepção da família sobre sua presença em uma unidade de terapia intensiva pediátrica e neonatal. Rev Esc. Enferm USP.43 (3): 630-638.

Moons P., De Geest S. \& Budts W (2002). Comprehensive care for adults with congenital heart disease: expanding roles for nurses. Eur J Cardiovasc Nurs. 1(1): $23-28$

Newburger J. W., Jonas R. A., Wernovsky G., Wypij D., Hickey P. R., Kuban K. C., et al. (1993). A comparison of the perioperative neurologic effects of hypothermic circulatory arrest versus low-flow cardiopulmonary bypass in infant heart surgery. N Englan J Med. 329(15): 1057-1064.

Oster M.E., Lee K. A., Honien M. A., Riehle-Colarusso T., Shin M. \& Correa A. (2013). Temporal trends in survival among infants with critical congenital heart defects. Pediatrics p. 131(5).

Pilz E. M. L. \& Schermann L. B. (2007). Determinantes biológicos e ambientais no desenvolvimento neuropsicomotor em uma amostra de crianças de Canoas/RS. Ciência \& Saúde Coletiva.12(1): 181-190.

Rappaport L. A., Wypij D., Bellinger D. C., Helmers S. L., Holmes G. L., Barnes P. D., et al. (1998). Relation of seizures after cardiac surgery in early infancy to neurodevelopmental outcome. Boston Circulatory Arrest Study Group. Circulation.97 (8): 773-779.

Ribeiro N. R. R. A família enfrentando a doença grave da criança. In: Elsen I., Marcon S. S. \& Silva M. R. S da (2002) editors. O viver em família e sua interface com a saúde e a doença. Maringá: Eduem; 199-220.

Sable C., Foster E., Uzark K., Bjornsen K., Canobbio M., Connolly H., et al. (2011). American Heart Association Congenital Heart Defects Committee of the Council on Cardiovascular Disease in the Young, Council on Cardiovascular Nursing, Council on Clinical Cardiology, and Council on Peripheral Vascular Disease. Best practices in managing transition to adulthood for adolescents with congenital heart disease: the transition process and medical and psychosocial issues: a scientific statement from the American Heart Association. Circulation. 123(13): 1454-85.

Santiago A. L (2011). Entre a saúde mental e a educação: abordagem clínica e pedagógica de sintomas na escola nomeados por dificuldades de aprendizagem e distúrbios de comportamentos. In: Santiago A. L. \& Campos R. H. J. Educação de crianças e jovens na contemporaneidade. Belo Horizonte: PUCMINAS; 201. 93-99.

Silva J. (2006) Avaliação e intervenção motora em crianças portadoras de cardiopatia congênita [dissertação]. Florianópolis: Universidade do Estado de Santa Catarina.

Silva A. M., Vaz C., Areias M. E. G. \& Vieira D. (2011) Quality of life of patients with congenital heart diseases. Cardiol Young. 21(6): 670-6.

Snookes S. H., Gunn J. K., Eldridge B. J., Donath S. M., Hunt R. W., Galea M. P., et al. (2010). A systematic review of motor and cognitive outcomes after early surgery for congenital heart disease. Pediatrics. 125(4): 818-27.

Uzark K., Lincoln A., Lamberti J. J., Mainwaring R. D., Spicer R. L. \& Moore J. W. (1998). Neurodevelopmental outcomes in children with Fontan repair of functional single ventricle. Pediatrics. 101(4): 630-3.

Williams P. G., Holmbeck G. N., \& Greenley R. N. (2002). Adolescent health psychology. J Consult Clin Psychol. 70(3): 828-42. 